\title{
Rencontre avec l'Autre lors du stage : utilisation du récit de pratique avec des étudiants en travail social et en ergothérapie
}

TÉTREAULT, Sylvie Haute école spécialisée de Suisse occidentale

LEANZA, Yvan

Université Laval, École de psychologie

GULFI, Alida ${ }^{1}$

Haute école spécialisée de Suisse occidentale

BÉTRISEY, Carine ${ }^{2}$

Haute école spécialisée de Suisse occidentale

KÜHNE, Nicolas

Haute école spécialisée de Suisse occidentale

BRISSET, Camille ${ }^{3}$ Université de Bordeaux

SCHAER, Martine Haute école spécialisée de Suisse occidentale

\section{RÉSUMÉ}

Préparer les étudiants en travail social et en ergothérapie à exercer dans un contexte de diversité constitue un défi actuel majeur. Or, peu d'informations sont disponibles sur les retombées des stages et de la rencontre avec des personnes aux cadres de référence divers sur la compétence interculturelle. La présente étude vise à documenter la perception que les étudiants ont de leur pratique auprès de l'Autre durant leur stage, les apprentissages qu'ils pensent avoir réalisés et les changements qu'ils identifient dans leur manière d'appréhender la diversité. Quinze étudiants de Suisse romande ont réalisé un récit de pratique sur un évènement marquant en lien avec la rencontre interculturelle. L'analyse thématique des propos recueillis permet d'illustrer comment ces expériences de stage ont contribué au processus de transformation vers une pratique culturellement sensible.

Mots-clés : compétence interculturelle, diversité, formation, analyse thématique.

\begin{abstract}
\footnotetext{
${ }^{1}$ HES-SO, Haute école de travail social Fribourg | HETS-FR | Fribourg

2 HES-SO, Haute école de travail social et de la santé | EESP | Lausanne

3 Université de Bordeaux, Laboratoire de psychologie EA 4139
}

Preparing students in social work and occupational therapy to practice in a context of diversity is a major current challenge. However, little information is available on the consequences of fieldwork and the encounter with people with various reference frameworks on intercultural competence. The purpose of this study is to document the students' perception of their practice with the Otherness 
during their fieldwork, the learning they think they have achieved and the changes they identify in their approach to diversity. Fifteen students from the French-speaking part of Switzerland wrote a practical account of a major event in connection with the intercultural encounter. The thematic analysis of the comments gathered illustrates how these internship experiences contributed to the process of transformation into a culturally sensitive practice.

Keywords: intercultural competency, diversity, education, thematic analysis.

\section{INTRODUCTION}

Former des étudiants dans le domaine de la santé et des services sociaux représente une tâche à maintenir constamment à jour. Plusieurs auteurs (Fattebert, Rüegger et Salerno, 2005 ; Dogra, Reitmanova et Carter-Pokras, 2009 ; Donoso Brown, Muñoz et Powell, 2011 ; Hall et Theriot, 2016 ; Reyneke, 2017) estiment qu'avec l'augmentation de la diversité des populations dans la société actuelle, les futurs professionnels seront amenés à œuvrer de plus en plus auprès de personnes aux ancrages culturels divers, notamment lors des stages. Par appartenances culturelles, il est généralement question de systèmes de significations partagés par un même groupe au travers desquels les individus donnent du sens à leurs expériences, et grâce auxquels ils guident leurs interactions avec autrui (Murden et al., 2008 ; Kohli, Huber et Faul, 2010 ; Hall et Theriot, 2016). D'autres auteurs, comme Beagan (2015) et Reyneke (2017), précisent que la diversité culturelle ne doit pas être entendue uniquement en lien avec un contexte migratoire. Elle comprend toutes les formes d'identité sociale et biologique ou d'expériences communes comme l'ethnicité, le genre, l'orientation sexuelle, la classe sociale, la religion, l'affiliation politique, les capacités physiques ou psychologiques (Whiteford et Wright St-Clair, 2002 ; Kohli et al., 2010 ; Beagan, 2015 ; Reyneke, 2017). Lorsque ces identités et expériences diverses, parfois opposées les unes aux autres, se rencontrent sur le terrain, l'attitude des différents protagonistes peut être influencée (Murden et al., 2008 ; Kohli et al., 2010 ; Govender et al., 2017). Par exemple, Govender et ses collègues (2017) soutiennent que des incompréhensions langagières et culturelles peuvent mener à un manque d'adhésion du client à la prise en charge en ergothérapie. Ces auteurs ont interrogé des étudiants sur le déroulement de leur stage. Ces derniers exprimaient une frustration quant aux difficultés rencontrées lors de l'établissement d'un lien thérapeutique avec des personnes ayant d'autres normes et valeurs culturelles ou religieuses. Des chercheurs (Legault et Rachédi, 2008 ; Hall et Theriot, 2016) ont identifié les difficultés des intervenants lors de leurs échanges avec des individus aux références culturelles différentes des leurs. Ils rapportent le développement par ces professionnels de stéréotypes et d'interprétations erronées des intentions et des actes de l'Autre. Dans le cas présent, l'Autre est celui qui est perçu comme porteur d'une différence radicale remettant en question les compétences professionnelles (Bredendiek et Krewer, 2003 ; Fedor, 2014). Cette situation peut parfois altérer la qualité de l'intervention (Legault et Rachédi, 2008 ; Hall et Theriot, 2016). À la lumière de ces résultats, il s'avère pertinent de saisir la dynamique qui s'installe entre les différentes personnes lors de la formation pratique dans les domaines de la santé et du travail social.

Dans le contexte actuel, préparer les étudiants à une pratique culturellement sensible est devenu un des défis majeurs des établissements de formation professionnelle (Fattebert et al., 2005 ; Donoso Brown et al., 2011; Hall et Theriot, 2016). À ce propos, Beagan (2015) et Cai (2016) constatent que ce type de pratique vise à tenir compte des valeurs, attitudes et manières d'agir des utilisateurs de services, comme de celles des intervenants. Ces auteurs affirment que le but de cette approche est d'amener les professionnels à s'intéresser activement aux références culturelles diverses, ainsi qu'à d'autres besoins ou façons de vivre. Pour ce faire, l'intégration dans les cursus 
de contenus sur la diversité et les interactions avec des personnes ayant un bagage culturel différent est fortement recommandée, que ce soit en travail social (Kohli et al., 2010 ; Hall et Theriot, 2016) ou en ergothérapie (Murden et al., 2008 ; Donoso Brown et al., 2011). De nombreux travaux mettent en évidence l'importance pour les étudiants de prendre conscience des changements d'attitude et de comportement qui peuvent survenir chez eux, suite aux contacts avec une clientèle diversifiée (Fattebert et al., 2005 ; Murden et al., 2008 ; Kohli et al., 2010 ; Donoso Brown et al., 2011 ; Hall et Theriot, 2016). En outre, ces études confirment la nécessité de développer une compétence interculturelle. Cette dernière consiste à mobiliser des connaissances et à utiliser des outils permettant la mise en place d'interventions respectueuses et adaptées aux besoins spécifiques des personnes accompagnées (Kohli et al., 2010 ; Cai, 2016 ; Hall et Theriot, 2016). Néanmoins, ces recommandations de considérer le cadre de référence de l'Autre n'explicitent pas toujours les modalités de mise en pratique (Bhui et al., 2007 ; Dogra et al., 2009 ; Donoso Brown et al., 2011). Même s'il existe des standards et des lignes de conduite établies, leur réalisation par des techniques concrètes de formation à la diversité et à la sensibilité interculturelle reste peu explorée en travail social et en ergothérapie (Fattebert et al., 2005 ; Kohli et al., 2010 ; Donoso Brown et al., 2011 ; Hall et Theriot, 2016). Différentes méthodes pédagogiques sont utilisées lors de la formation professionnelle (présentations orales, vidéos, études de cas, jeux de rôle, expériences sur le terrain, travail réflexif, etc.), mais leur efficacité est peu documentée (Murden et al., 2008 ; Kohli et al., 2010 ; Donoso Brown et al., 2011). Par exemple, Murden et ses collaboratrices (2008) rapportent que les étudiants en ergothérapie jugent leur formation dans le domaine interculturel inadéquate ou insuffisante, notamment par manque d'exemples concrets de la pratique professionnelle au contact de la diversité. Pourtant, l'expérience acquise auprès d'une clientèle hétérogène est considérée comme un facteur de croissance de la compétence interculturelle (Murden et al., 2008 ; Kohli et al., 2010 ; Donoso Brown et al., 2011 ; Hall et Theriot, 2016). De ce fait, augmenter le nombre de ces expériences constitue un moyen de former les étudiants à ce type d'interventions. Dans ce sens, les stages sont l'occasion de réaliser des rencontres avec l'Autre. Par ailleurs, les écrits scientifiques notent l'importance de l'encadrement des étudiants lors de la pratique en contexte pluriculturel, afin d'offrir un milieu d'apprentissage sécurisant (Kohli et al., 2010 ; Hall et Theriot, 2016 ; Reyneke, 2017). À ce propos, Reyneke (2017) déclare que les étudiants doivent pouvoir exprimer leurs émotions (colère, anxiété, ambivalence, etc.) en toute confiance, par la présence d'enseignants veillant à les accueillir, sans les juger ou les pénaliser. Lors des stages, l'encadrement par des superviseurs adéquatement formés paraît essentiel, tout comme lors des séances en classe (Reyneke, 2017).

Le modèle de la niche d'activités professionnelles (NAP) développé par Leanza (2011) a été choisi comme cadre théorique pour la présente recherche. La NAP explore les différentes dimensions de l'expérience de rencontre avec la diversité culturelle chez les professionnels en devenir, et ce, dans un contexte de stage. Leanza (2011) définit trois dimensions dans le modèle de la NAP : (1) le contexte de l'activité professionnelle ; (2) les ethnothéories ou représentations des professionnels ; (3) les pratiques elles-mêmes. Le futur professionnel se situe au cœur de cette niche ou de cet environnement de travail (matériel et cognitif). Avec cette dynamique, il est possible d'évaluer comment chacun vit et se positionne par rapport à ce que lui prescrit sa NAP, à savoir son rapport au contexte, aux ethnothéories, à sa propre pratique, en plus de son rapport aux individus ayant des ancrages culturels divers (Leanza, 2011).

L'intérêt de la présente étude porte sur la manière dont les étudiants en travail social et en ergothérapie perçoivent leurs comportements professionnels et les changements qui s'opèrent en eux lors de rencontres avec des personnes qu'ils considèrent comme étant porteuses d'une différence. Plus particulièrement, cet article vise à répondre aux trois questions suivantes : (1) 
Comment les étudiants perçoivent-ils leurs interventions professionnelles en interaction avec l'Autre durant leur premier stage ? (2) Quels apprentissages considèrent-ils avoir réalisés lors de ces interactions ? (3) Quels changements identifient-ils dans leur manière d'appréhender la rencontre avec l'Autre suite à ce stage ?

\section{MÉTHODOLOGIE}

En vue de documenter l'interaction avec la diversité culturelle chez les étudiants en travail social et en ergothérapie durant leur stage, un devis qualitatif incluant des récits de pratique a été privilégié. Il faut préciser que cette étude s'intègre dans un projet plus vaste, qui implique un suivi longitudinal des étudiants sur trois années de formation (Tétreault, Gulfi et Kühne, Projet FNS, 2016-2019).

\section{Sélection des participants}

Les participants ont été recrutés parmi les étudiants en travail social et en ergothérapie de deux Hautes écoles de Suisse romande. Selon les standards éthiques de la recherche qualitative en Suisse, l'accord des responsables de programme des deux écoles a été obtenu avant de contacter ces étudiants (01-05-2016). Les participants ont également signé un formulaire de consentement au début de cette étude. Les 16 personnes ayant pris part à la première collecte de données (an 1) ont été recontactées par courriel lors de leur deuxième année d'études afin de participer à un entretien sous forme de récit de pratique (an 2). Celles qui étaient intéressées ont reçu un feuillet d'information sur la manière de préparer le récit de pratique. Seule cette deuxième récolte de données est considérée dans la présente étude.

\section{Outils et procédure de collecte d'information}

Le guide d'entretien semi-directif se base sur le modèle du récit de pratique (Bertaux, 1997 ; Audet, 2008). Cette forme d'entrevue permet aux participants de se remémorer un évènement significatif (ici, la rencontre avec l'Autre), d'approfondir leur réflexion à son propos, d'en expliciter les conséquences et le sens qui lui est attribué. Par cette méthode d'autoréflexion, il est possible d'accéder « de l'intérieur » à une situation problématique que les étudiants ont eu à gérer. Le guide d'entretien permet aussi de conserver une structure similaire entre les informateurs-clés (Miles, Huberman et Saldaña, 2014). Constitué de questions ouvertes, il a été construit sur la base du cadre théorique de la niche d'activités professionnelles (NAP) (Leanza, 2011). L'équipe de recherche a discuté et validé ce guide, avant de l'utiliser.

Durant l'entretien, l'étudiant aborde un évènement significatif de son choix, qui implique une personne porteuse de différences et qui s'est produit durant son premier stage. L'expérience doit avoir suscité une réaction marquante, un examen plus approfondi d'une idée, voire une remise en question de ses représentations et de ses pratiques. En ce sens, elle est susceptible d'orienter ses choix ou ses comportements professionnels, et possiblement d'entraîner un changement de valeurs ou d'opinions. Une fois que l'étudiant a choisi une expérience, il lui donne un titre et en décrit le contexte. Le déroulement de l'évènement est ensuite détaillé, de même que les pensées qu'il a suscitées et les émotions qui ont été ressenties sur le moment. L'étudiant explique le sens attribué aux comportements de chacun et les raisons de ces réactions. Les éléments positifs, les améliorations possibles et les apprentissages réalisés par l'étudiant suite à cette expérience sont aussi spécifiés, tout comme la manière dont cette interaction l'a touché. Puis, la réflexion après coup par rapport à l'événement, les émotions et les pensées qui y sont associées sont discutées. Le participant examine les retombées de l'expérience décrite et la manière dont celle-ci a modifié sa façon d'aborder les personnes ayant un bagage culturel différent. II précise les apprentissages 
réalisés en lien avec sa future profession. Les entretiens, effectués dans un lieu choisi par l'étudiant (école, domicile ou lieu public), ont duré entre 30 et 60 minutes. Ils ont été enregistrés, puis retranscrits intégralement au fur et à mesure de la récolte des données. Pour chaque entretien, un résumé a été produit, puis envoyé au participant pour validation.

\section{Méthode d'analyse des données}

Suite à une double vérification des transcriptions des entretiens, une analyse thématique est utilisée pour traiter les propos recueillis (Miles et al., 2014). Une première lecture flottante est effectuée afin de prendre connaissance du contenu du discours des participants et de s'en imprégner (Bardin, 2013). À partir des trois questions de recherche, des regroupements d'idées récurrentes sont ensuite réalisés (Thomas, 2006 ; Miles et al., 2014) et codés à l'aide du logiciel NVivo@. Selon Miles et ses collaborateurs (2014), les thèmes ainsi obtenus représentent des codes explicatifs qui identifient un " pattern » d'idées. Cette codification constitue le moyen de synthétiser et condenser les propos des participants. Dans un processus progressif et itératif, une grille thématique regroupant des thèmes et des sous-thèmes est construite et raffinée, en réponse aux trois questions de recherche. L'analyse des transcriptions se fait par une seule personne, puis est vérifiée par une seconde, ce qui favorise la validité interévaluateur (Miles et al., 2014). L'ensemble du processus et de ses résultats est discuté à plusieurs reprises entre les chercheurs. Diverses mesures ont été utilisées afin d'augmenter la scientificité des résultats (Drapeau, 2004 ; Careau et Vallée, 2014). Tout d'abord, un journal de bord, colligeant les décisions et les évènements significatifs durant les entretiens, a été tenu tout au long de l'étude. Le contenu de ce journal sert à mettre en contexte la réalisation des rencontres avec les informateurs-clés. II faut préciser qu'une seule personne a effectué l'ensemble des entretiens afin de conserver une stabilité et une homogénéité du processus de collecte des informations. II faut noter également que cette personne n'enseigne pas dans les deux programmes d'études ciblés, ce qui permet de réduire le biais de désirabilité sociale. Par ailleurs, la validation des synthèses des verbatims par les participants contribue à la crédibilité des données obtenues. Enfin, l'équipe de recherche s'est consultée régulièrement pour échanger sur les analyses en cours (cohérence interne).

\section{RÉSUltats}

Afin de contextualiser les récits de pratique, une description des participants et de leurs stages est présentée. Les autres résultats sont formulés en fonction des trois questions de recherche : (1) la représentation qu'ont les étudiants de leurs interventions professionnelles en interaction avec l'Autre ; (2) les apprentissages qu'ils estiment avoir réalisés suite à ces rencontres interculturelles ; (3) les changements qu'ils perçoivent dans leur manière d'appréhender l'Autre durant leur pratique professionnelle.

\section{Caractéristiques des participants et de leurs stages}

En tout, 15 étudiants ont accepté de participer à l'entretien, soit 9 (60\%) en travail social et 6 (40\%) en ergothérapie. II s'agit de 13 femmes (87\%) et 2 hommes (13\%), âgés de 19 à 42 ans (moyenne d'âge de 24 ans, écart-type $=5,7)$. À une exception près, tous sont nés en Suisse. Six d'entre eux (40\%) possèdent une double nationalité. Huit étudiants (53\%) ont fait une ou plusieurs formations professionnelles avant celle suivie au moment de l'entretien. Aucune information sur leur état civil ou leur statut socioéconomique n'a été recueillie.

En ce qui concerne les caractéristiques des stages, parmi les neuf étudiants en travail social, quatre ont accompli leur pratique de terrain en tant qu'éducateurs sociaux dans des foyers d'accueil, quatre 
autres comme assistants sociaux et une dernière en tant qu'animatrice socioculturelle. Ils ont accompagné des personnes connaissant diverses problématiques (itinérance, rupture familiale ou sociale, parcours migratoire, situation de dépendance, etc.). Pour leur part, les six étudiants en ergothérapie sont allés dans différents types d'établissements (hôpitaux, cliniques publiques ou privées, centres médico-sociaux). Ils sont intervenus auprès de personnes ayant divers problèmes de santé physique ou mentale. II faut préciser que 3 des 15 informateurs-clés ont effectué leur premier stage à l'étranger.

\section{Perception de ses propres interactions professionnelles avec l'Autre}

La première question de recherche explorée cible la perception que les étudiants ont de leurs interactions professionnelles avec des personnes aux ancrages culturels divers. L'analyse de leurs propos au sujet d'un évènement significatif qu'ils ont choisi de raconter fait ressortir trois " patterns récurrents ", ainsi nommés par Miles et al. (2014), soit la nécessité de : (1) prendre conscience de ses compétences et de ses limites ; (2) s'adapter à l'Autre, en tenant compte de ses différences ; (3) apprendre à s'ajuster aux règles de fonctionnement institutionnelles.

\section{Prendre conscience de ses compétences et de ses limites}

Les participants relèvent plusieurs compétences professionnelles qu'ils estiment posséder et qu'ils considèrent comme ayant facilité leur intervention lors du stage. II s'agit notamment d'établir le contact avec l'Autre et d'ajuster son intervention à ses caractéristiques. De plus, ils rapportent la nécessité d'adopter une attitude de non-jugement et de manifester de l'empathie. Par exemple, une étudiante en travail social explique qu'elle a dû se préparer pour intervenir auprès d'une personne en situation de dépendance, et même tenter d'anticiper des comportements d'opposition : «Quand elle est arrivée, donc moi, j'étais préparée à ce qu'elle me demande une avance de 30 francs, à la base, et puis j'étais préparée à lui dire non. " En repensant à cette situation, elle se félicite d'avoir su tenir sa position, malgré la forte insistance de son interlocutrice. Quelques participants disent avoir éprouvé des difficultés dans l'interaction avec l'Autre. Souvent, un sentiment d'impuissance ou d'échec est évoqué par ceux-ci devant des situations complexes. Ainsi, une étudiante en travail social, qui se décrit comme hypersensible, raconte s'être trouvée désemparée en écoutant le témoignage d'une personne ayant vécu une expérience de guerre :

Donc, elle s'est tout d'un coup ouverte, cette dame, sur son parcours... enfin, sur le conflit qu'elle a vécu en [nom du pays]. Et puis c'était assez fort en émotion parce qu'elle s'est mise à pleurer. Alors du coup, pour moi, c'était un peu : «Oh mon Dieu! » Je tombais dans la sympathie. Alors que pas du tout, on a appris à être empathique.

Une autre difficulté fréquemment énoncée dans les entretiens concerne l'interprétation parfois faussée d'évènements lors du stage. À ce propos, plusieurs participants notent que leur jugement a pu être influencé de différentes manières par des caractéristiques de l'Autre ou le contexte de travail (c'est-à-dire contexte culturel, vécu secondaire de la situation, informations biaisées). Par exemple, une étudiante en ergothérapie remet en question son interprétation d'une situation à domicile au cours de laquelle la conjointe ne s'est pas impliquée (aucun commentaire, question ou intérêt de sa part). II s'agissait d'un couple mixte et l'étudiante s'exprime sur le sujet :

[...] c'est le comportement lui-même qui m'a interloquée. Est-ce que pour ça, je l'ai rattaché après à la nationalité en me disant : «Ah, mais peut-être c'est un mariage 
arrangé, quelque chose comme ça... »? Peut-être que pour une personne européenne, je ne me serais pas posé de questions beaucoup plus loin.

Elle estime après coup que cela a affecté sa «neutralité » professionnelle et contribué à une perception négative de la situation.

\section{S'adapter à l'Autre, en tenant compte de ses différences}

Lorsque l'Autre adopte un comportement imprévu, il arrive que les participants se trouvent démunis et doutent de la réaction à avoir. Pour quelques-uns, l'adaptation s'est réalisée avec le temps et l'expérience. Par exemple, une participante évoque les ajustements qu'elle a dû faire lors de son stage avec des personnes qui présentaient des problèmes de santé mentale : «[...] devoir constamment adapter, bien, ce que je dis, et comment je le dis, et comment je l'explique, et comment je vais réfléchir, pour ces gens-là. [...] C'est plus là que ça me fait changer. » De son côté, une étudiante en travail social dit avoir modifié son intervention en fonction des caractéristiques qu'elle attribue à l'Autre. Elle affirme mobiliser différentes ressources selon le niveau d'éducation des personnes qu'elle accompagne :

Genre, quand je m'entretiens avec un [nationalité], je me dis : «Ah, bien il est sûrement... il a eu de l'éducation dans son pays. C'est quelqu'un qui parle le français et qui sait plein de choses, qui est motivé. " Tandis que quand je rencontre un [autre nationalité], c'est vrai que je vais plutôt facilement me dire : "C'est possible que cette personne soit moins motivée parce qu'il n'a pas eu forcément une scolarité grandiose dans son pays. II n'a pas continué les études. 》

Puis, la jeune femme ajoute qu'elle cherche à développer les ressources internes des personnes ayant un niveau d'éducation peu élevé. Pour les bénéficiaires plus éduqués, elle a tendance à leur faire connaître des ressources externes, puisque selon elle, ces gens sont capables de réaliser les démarches eux-mêmes.

Parfois, le niveau de langage utilisé lors de l'intervention doit être ajusté, en particulier auprès de clientèles qui ne maîtrisent pas le français. Un participant donne un exemple vécu lors de son stage comme éducateur social auprès de jeunes migrants. Lorsqu'il se retrouve avec un groupe d'individus du même pays, ceux-ci discutent parfois entre eux dans leur langue. L'étudiant estime que cette situation crée une distance, voire un malaise dans ses interactions avec les Autres, par manque de compréhension de ce qui se dit. Dans le même sens, une étudiante en ergothérapie a dû utiliser une langue qu'elle pensait maîtriser, lors de ses interactions avec une personne germanophone ayant des séquelles d'un AVC. Elle formule ainsi ce qu'elle a ressenti sur le moment :

Bien d'abord, je me suis dit que je n'étais vraiment pas en train de l'aider avec mon allemand un peu médiocre. Ça devait déjà être assez difficile pour lui d'essayer de comprendre quelqu'un qui parle correctement. Alors je n'étais vraiment pas en train de l'aider.

La participante confie avoir été en difficulté lorsqu'elle a tenté de s'expliquer en allemand. Devant le même type de situation, mais adoptant une stratégie différente, un étudiant en ergothérapie a fait appel à une autre personne. Cette dernière a agi comme interprète lors d'échanges avec une patiente allophone en isolement dans sa chambre. Le jeune homme s'explique :

Je lui dis qu'elle ne peut pas sortir. Elle me regarde. Je n'étais pas sûr d'avoir compris... enfin, qu'elle ait compris. Là, j'ai profité qu'il y ait un concierge qui était là pour lui demander s'il parlait portugais parce qu'il me semblait qu'il... Et c'est lui 
qui m'a servi d'interprète pour... enfin, je lui ai transmis la consigne qu'elle n'avait pas à sortir. Et c'est lui qui [...] m'a traduit qu'elle attendait, en fait, le physiothérapeute qui devait venir.

Le participant avoue qu'il ne s'est pas senti à l'aise dans cette situation.

\section{Apprendre à s'ajuster aux règles de fonctionnement institutionnelles}

Les étudiants expriment des difficultés vécues en lien avec l'institution dans laquelle leur stage s'est déroulé (mandat, règles, pratiques prescrites, etc.). Parfois, ils se retrouvent tiraillés entre l'application des règlements et leur conduite spontanée. À ce propos, une participante signale qu'elle a eu du mal à ne pas s'impliquer émotionnellement, à conserver une distance professionnelle. Elle trouve pénible d'être témoin des démarches infructueuses de réinsertion des personnes qu'elle accompagne. L'étudiante en travail social confie son désarroi :

Et puis ça, ça me révolte aussi un peu contre la fonction de l'assistante sociale, finalement. On est des assistants sociaux, mais on ne peut pas faire notre boulot de travailleur social. On est fonctionnaire de l'État de [région] plus que travailleur social, j'ai l'impression. On dépend du budget. On dépend de ce qu'on peut offrir aux bénéficiaires. On ne peut pas forcément toujours aller dans leur sens.

Dans ces conditions, les participants mettent en évidence le besoin d'affiner leurs compétences, afin d'éviter de se retrouver démunis face à l'Autre et d'éprouver un sentiment d'impuissance. Ils disent vouloir mieux connaître les ressources à leur disposition pour aider les bénéficiaires. Souvent, ceux qui ont fait leur stage à l'étranger ont expérimenté d'autres types d'institutions et des pratiques différentes auxquelles ils ont dû s'adapter. Dans ce sens, une jeune femme raconte comment elle a dû faire face à l'imprévisibilité de ses tâches et aux caractéristiques de son milieu de stage dans un autre pays :

Bien je sais que durant cette semaine, je me dis : «Bien j'écrirai ces rapports-là, mais je ne peux pas dire quel jour et à quelle heure parce qu'il y a tout le temps des changements de programme. " Donc j'ai vraiment changé ma manière de penser.

Selon elle, la cohésion d'équipe est plus valorisée dans ce pays qu'en Suisse. Également lors d'un stage à l'étranger, une autre étudiante en travail social décrit ses difficultés à s'adapter aux règles de fonctionnement d'une institution quant à la nutrition des jeunes enfants. Toutefois, après un long travail de réflexion, elle conclut :

[...] le [pays], oui, c'est différent de nous. Donc ça arrive peut-être plus souvent de rencontrer des situations où les comportements des gens diffèrent des nôtres, non ? Quand même. Mais je fais la réflexion que ça pourrait m'arriver dans tout lieu de formation ou de travail [...].

Pour elle, les différences culturelles peuvent découler de beaucoup d'éléments (âge, état de santé, expériences de vie, etc.) qui ne dépendent pas uniquement du pays dans lequel le stage se déroule.

\section{Apprentissages réalisés suite au contact avec l'Autre}

La deuxième question de recherche cible les apprentissages identifiés par les étudiants comme résultant de leur rencontre avec l'Autre durant le stage. Deux constats se dégagent, soit le besoin : 
(1) d'acquérir des connaissances sur d'autres groupes culturels ; (2) de développer des outils pour la pratique en contexte de diversité.

\section{Acquérir des connaissances sur d'autres groupes culturels}

Lors des stages, beaucoup ont côtoyé de nouvelles clientèles, expérimentant parfois l'influence que les appartenances culturelles peuvent avoir sur les comportements et les croyances des individus. Plusieurs informateurs-clés constatent que la diversité n'entrave pas forcément l'intervention, mais demande d'observer davantage les modes de fonctionnement de l'Autre pour essayer de les comprendre. C'est notamment le cas de deux étudiants en travail social dont la formation pratique s'est déroulée auprès de personnes ayant un parcours migratoire. Ainsi, l'un d'eux fait le point sur ses apprentissages à propos du fonctionnement social d'un groupe culturel : “ Et puis après, bien, sur la culture [pays], justement, tout ce contrôle social, les choses comme ça, bien c'est vrai que je connais mieux maintenant. " II révèle avoir compris pourquoi certains développent une perception négative des institutions étatiques selon leur vécu dans leur pays d'origine (oppression, corruption).

\section{Développer des outils pour la pratique en contexte de diversité}

Des informateurs-clés disent avoir réalisé l'importance d'être sensibles et ouverts aux valeurs différentes de leur suite aux stages. Ils ont dû développer des outils ou manières d'agir envers l'Autre. Par exemple, une participante énonce les précautions qu'elle a dû prendre lors de son intervention auprès de personnes d'une religion différente de la sienne :

Quand on sait qu'on va aller dans une partie [religion], on ne va pas faire exprès de s'habiller avec des T-shirts trop courts ou bien... [...] On respecte, on les respecte, en fait. Parce que s'ils voient qu'on les respecte, eux aussi, ils feront un effort envers nous.

De même, une étudiante en ergothérapie détaille comment elle a appris à se comporter avec des individus qui présentent des problèmes de santé mentale : "C'est clair que ça me force à apprendre, là, oui, à désamorcer des situations, à comment reprendre quelqu'un et le recadrer, mais de la manière appropriée, au bon moment [...]. » Par ailleurs, la plupart des étudiants, qui ont côtoyé des clientèles allophones, ont appris à gérer cette différence par l'adaptation de leur communication, comme l'illustre cette jeune femme : «[...] j'ai appris à comment plus utiliser le mime, et puis les gestes aussi. Parce que les autres patients que j'ai, finalement, ils comprennent bien les consignes. " Lors de séances en ergothérapie, elle a utilisé les gestes pour indiquer les exercices à accomplir. Dans un autre domaine, une étudiante en travail social a dû s'adapter aux interactions avec des personnes en situation de toxicomanie :

[...] je me suis rendu compte quand même que l'argent, il influence quand même vachement les rapports entre nous, les travailleurs du [institution], et puis les personnes qui sont suivies. Parce que si on fixe un rendez-vous avec une personne au moment où on doit lui remettre son argent, bien en fait, ça n'a pas de sens. [...] Donc l'argent, il a quand même une place très très centrale dans leur vie, due aux consommations.

Cette jeune femme mentionne clairement avoir pris conscience de la manière dont le désir de consommer et le besoin d'argent influencent la relation d'aide. 


\section{Changements perçus dans la manière d'appréhender l'Autre}

La troisième question de recherche porte sur les changements perçus par les étudiants suite au stage dans leur manière d'appréhender les rencontres interculturelles. Trois changements ressortent, soit : (1) la prise de conscience de ses préjugés et représentations erronées de l'Autre ; (2) l'adoption d'une représentation plus juste de la profession en contexte de diversité ; (3) l'engagement dans un processus de transformation.

\section{Prise de conscience de ses préjugés et représentations erronées de l'Autre}

Quelques informateurs-clés affirment avoir pris conscience du fait que leurs représentations de l'Autre et leurs pratiques professionnelles sont parfois biaisées. Par exemple, l'appréciation d'une situation peut être influencée par le discours des autres membres de l'équipe, en particulier si l'étudiant ne participe pas directement à l'intervention (rôle d'observateur). C'est le cas de cette jeune femme qui parle de son sentiment d'insécurité face à un bénéficiaire ayant des troubles de la santé mentale. Elle constate que cette peur était construite sur la base des comptes rendus de ses collègues lors de réunions d'équipe. C'est pourquoi elle admet ceci :

[...] il faut aussi se faire sa propre perception des personnes, et non pas que ce soit ce que disent les autres qui crée ma perception. Alors il y aura de toute façon une partie de ça, mais aussi créer vraiment ma propre perception, et justement, analyser la personne, ses comportements, voir pour mieux la comprendre, mieux la connaître.

Quelquefois, la rencontre avec l'Autre peut aussi casser des stéréotypes. Une participante rapporte qu'elle a pu se faire sa propre idée sur les personnes en situation de toxicomanie lors de son stage : «Enfin, oui, ils dégagent une image assez négative, alors qu'en fait, ce sont des gens, pour la plupart, qui n'ont juste pas eu de chance et puis qui ont commencé à consommer. " La jeune femme affirme avoir changé de point de vue sur ces personnes. De même, une étudiante en travail social s'exprime ouvertement sur ses préjugés : « [...] j'avais plein de préjugés, des choses par rapport à l'Autre, à ces cultures du Moyen-Orient, où j'étais plutôt réticente par rapport... enfin, à l'inconnu. [...] Alors que pas du tout... enfin, au contraire. "Suite à ses interactions durant le stage, cette participante avoue avoir modifié son jugement envers ces groupes culturels. Néanmoins, certains étudiants observent que le fait d'interagir avec l'Autre peut aussi favoriser parfois l'émergence de préjugés, que ce soit vis-à-vis du fonctionnement de la personne accompagnée ou de l'institution dans laquelle le stage se déroule. Par exemple, une étudiante en ergothérapie est décontenancée du comportement de non-collaboration d'une patiente dont le pouce a été amputé :

Bien je me suis dit : « C'est quand même bizarre parce que... enfin, le pouce, on en a quand même vraiment besoin. Donc si on dit qu'il faut mettre une crème pour cicatriser, c'est quand même important. » Oui, ça m'a franchement étonnée qu'elle n'ait pas écouté le conseil.

Elle avoue avoir trouvé « un peu bizarres » les croyances de la patiente quant à la cicatrisation. Avec l'amorce d'une réflexivité sur leurs propres biais d'interprétation lors du récit de pratique, des étudiants disent s'apercevoir que leurs attitudes professionnelles évoluent, changent et se transforment. Plusieurs insistent sur le besoin de ne pas porter de jugement sur les comportements des personnes aux références culturelles diverses, comme l'exprime cette jeune femme : « Je pense que je vais essayer d'être... enfin, de mettre un peu de côté ce que moi, je pense, pour me concentrer sur comment la personne voit les choses. Enfin, d'être dans l'empathie, quoi. » Dans le même sens, une étudiante en travail social conclut : 
Alors pour moi, c'est une expérience qui est de toute manière formatrice. Comment ça va se passer par la suite avec d'autres personnes d'une autre culture, je pense que je vais continuer à essayer de les apprendre, d'apprendre à connaître leur monde parce que je trouve que c'est vraiment intéressant. Et en apprenant à connaître leur monde, bien on peut aussi à nouveau mieux les accompagner.

Cette participante met en avant l'importance de l'observation de l'Autre et de la compréhension de son fonctionnement, tout cela afin de s'aider dans son développement professionnel.

\section{Adoption d'une représentation plus juste de la profession en contexte multiculturel}

Plusieurs des personnes rencontrées dans le cadre de cette étude rapportent avoir modifié leur façon de se représenter leur profession et envisagé différemment les retombées de leurs interventions auprès de personnes aux ancrages culturels divers. Suite à un stage à l'étranger, une étudiante en travail social déclare : « Donc je pense qu'en fait, j'ai relativisé mon intervention. J'arrive à me dire que je ne suis pas toute-puissante [...]. " Quelques-uns soutiennent avoir aussi imaginé le travail social de manière idéaliste. Or, ils confirment par leurs propos que la pratique est très différente de ces conceptions préalables. Ainsi, le stage les amène à avoir une idée plus juste de leur profession. Par exemple, l'impossibilité de forcer la personne à agir demande d'adopter une attitude d'acceptation envers l'Autre, tel que l'illustre cette étudiante en travail social :

On ne peut jamais tout savoir sur une famille. [...] Ils ne sont pas obligés de tout nous dire. [...] on ne peut pas les obliger, même que des fois, on aurait besoin [de ces informations] parce que ce serait beaucoup plus facile [...].

Elle indique qu'il faut s'ajuster à la situation, sans devenir intrusif. Néanmoins, des participants signalent qu'il n'est pas toujours possible de respecter le point de vue de l'Autre et ses décisions. Dans ce sens, une étudiante en travail social revient sur son expérience avec un père d'origine asiatique dont les enfants sont placés en foyer et qui fait souvent référence aux normes de sa culture d'origine :

[...] avant cette situation, j'aurais pensé que, un peu, assez naïvement, que bien la culture des gens, c'est quelque chose qu'on ne peut pas changer et puis qu'on doit comprendre, et puis qu'on doit faire avec. Et puis maintenant, avec cette situation, je me dis : «Bien là, il l'utilise quand même beaucoup pour répondre à plein de questions, mais pour ne pas aller plus loin dans ses réflexions. » Et que du coup, bien là, peut-être qu'on devrait aller au-delà de la culture et peut-être ne plus prendre ça en compte.

Selon elle, il faut parfois remettre en question les choix de l'Autre, même si celui-ci évoque sa culture comme point d'ancrage de son comportement.

\section{Engagement dans un processus de transformation}

Lorsqu'ils repensent à leurs interactions en contexte de diversité, les participants perçoivent souvent une transformation, un changement dans leur manière d'être et d'agir. Par exemple, quelques-uns relèvent la nécessité d'observer et de mieux connaître l'Autre, de savoir quelles sont ses habitudes de vie et ses croyances, afin d'adapter l'accompagnement proposé. Dans ce sens, une étudiante en ergothérapie confie comment elle aurait pu améliorer son intervention auprès d'une femme ayant un parcours migratoire: " Je trouve qu'on devrait, oui, plus demander à la personne ce qu'elle a I'habitude de faire, qu'est-ce qu'elle croit qui peut aider, avant de vraiment lui soumettre quelque 
chose [...]. » Elle complète en rapportant la nécessité de chercher les raisons qui peuvent amener l'Autre à refuser une intervention, celles-ci étant parfois en lien avec des croyances individuelles. Cette ouverture d'esprit et cette capacité de non-jugement se sont développées chez plusieurs au contact de clientèles diversifiées, comme le montre cet autre témoignage d'une étudiante en ergothérapie :

[...] il faut vraiment faire avec les fonctionnements très différents des personnes et s'y adapter au mieux, parce que, bien, finalement, voilà, la personne, elle évolue dans un environnement qui a son fonctionnement, et qu'il faut respecter ça, même si ça nous paraît complètement saugrenu.

Elle estime qu'il faut de prendre du recul et remettre en question ses propres interprétations, le cas échéant.

En matière de communication, ceux qui ont interagi avec des personnes allophones constatent que les difficultés liées à la langue sont surmontables. En modifiant et en adaptant les moyens de communication utilisés, les échanges peuvent être facilités. Une étudiante en ergothérapie mentionne ceci : "Même si on n'a pas le mot en anglais, bien on peut, je ne sais pas, le dessiner ou vite chercher une traduction, ou le mimer, je ne sais pas. II y a toujours une manière de se faire comprendre. "Selon les propos recueillis, les expériences interculturelles vécues durant le stage ont permis d'acquérir une meilleure aisance dans les contacts professionnels avec l'Autre. À ce sujet, une participante détaille les modifications qu'elle pense mettre en place pour sa pratique avec des patients allophones : «[...] bien maintenant, je pense que j'essaierais plus directement d'aller dans la communication avec les gestes. [...] de montrer en même temps, un peu plus spontanément que là. " Dans le même sens, une étudiante en travail social revient sur sa première rencontre avec une personne ayant des troubles psychiques:

Donc non, moi, je trouve que ce stage m'apporte énormément. Je trouve que ce n'est pas toujours évident de tout qualifier et tout, mais au niveau de l'observation de ce que je fais, je me rends compte qu'après, aussi, mes interventions en tant que telles s'améliorent de semaine en semaine.

Grâce à ses expériences en stage, la jeune fille soutient qu'elle a acquis plus de tact et qu'elle est plus authentique avec les résidents de l'établissement où elle travaille, tout en sachant garder une certaine distance pour se protéger. De manière générale, les étudiants se sentent souvent plus en confiance par rapport à leurs interventions et aux outils qu'ils ont à leur disposition.

\section{Discussion}

Une des questions posées dans la présente étude vise à documenter la manière dont les étudiants en travail social et en ergothérapie perçoivent leur pratique professionnelle lors d'interactions avec des personnes aux références culturelles différentes des leurs. L'analyse des 15 entretiens met en évidence la prise de conscience à la fois de compétences et de limites en lien avec une pratique interculturelle. D'un côté, les participants disent posséder des aptitudes comme l'empathie et des capacités d'adaptation et d'anticipation facilitant l'intervention dans un contexte de diversité. Mais d'un autre côté, ils ressentent parfois un sentiment d'échec ou d'impuissance lors de situations complexes. Selon Côté (2014), ce ressenti peut résulter d'un écart de perception et d'interprétation au sein de la relation professionnel-patient. II arrive alors que cette frustration mène à la production ou au renforcement de jugements négatifs chez l'intervenant (Côté, 2014). Des participants ont reconnu que leur compréhension des conduites de l'Autre a pu être influencée par des idées préconçues ou par le discours d'autres professionnels sur le terrain, ces constats concordant avec 
ceux de Doutrich et Storey (2004) auprès d'infirmières. Selon Reyneke (2017), cette prise de conscience est favorisée par le dialogue avec des personnes considérées comme porteuses d'une différence. Toutefois, il est essentiel que ces échanges se fassent dans un environnement sécurisant, étant donné le stress, l'angoisse, la frustration ou encore la culpabilité que la découverte de ces préjugés peut entraîner chez les étudiants (Doutrich et Storey, 2004 ; Kohli et al., 2010 ; Hall et Theriot, 2016 ; Reyneke, 2017). Plus précisément, Doutrich et Storey (2004) et Reyneke (2017) affirment que la présence de superviseurs formés à accueillir ces émotions sans jugement est essentielle.

Un deuxième résultat concerne la manière dont les participants disent s'être adaptés aux caractéristiques de l'Autre. Selon les propos obtenus, ces ajustements semblent se mettre en place avec le temps et l'expérience. Lorsqu'une personne ne maîtrise pas la langue française, les étudiants sont parfois déstabilisés, mais finissent par développer divers comportements d'adaptation. Donoso Brown et ses collaborateurs (2011) confirment cette situation et affirment que les habiletés de communication interculturelle s'améliorent par le contact direct avec la diversité. Pour faciliter les échanges, Govender et ses collaborateurs (2017) proposent plusieurs méthodes, comme le recours à un médiateur culturel ou un interprète, l'utilisation du langage non verbal, ou encore la recherche documentaire.

Un dernier résultat lié à la perception des pratiques professionnelles porte sur le défi que peut constituer l'ajustement aux règles de fonctionnement institutionnelles. Que ce soit en Suisse ou à l'étranger, les étudiants doivent évoluer dans un cadre qui leur prescrit des règles de conduite à respecter. Cela entraîne parfois de la frustration et un sentiment d'impuissance. À ce propos, Feize et Gonzales (2018) mettent en évidence des situations similaires dans l'enseignement de la sensibilité interculturelle. Leur étude atteste que les règles imposées par les établissements peuvent entrer en contradiction avec la volonté de s'adapter à la culture de l'Autre.

Les apprentissages perçus par les étudiants suite au stage correspondent à la deuxième question de recherche. D'abord, les informateurs-clés rapportent que le fait de côtoyer de nouvelles clientèles a contribué à augmenter leurs connaissances sur d'autres groupes culturels. À ce sujet, certains expliquent qu'ils ont dû davantage observer les manières d'agir de l'Autre pour les comprendre et s'y adapter. II s'agit là d'un point essentiel d'une pratique culturellement sensible, comme l'ont affirmé de nombreux auteurs (Murden et al., 2008 ; Kohli et al., 2010 ; Cai, 2016 ; Hall et Theriot, 2016 ; Reyneke, 2017).

Un deuxième constat lié aux apprentissages perçus par les étudiants concerne le développement de la sensibilité et de l'ouverture d'esprit. Cela a déjà été documenté dans d'autres travaux comme conséquence de la rencontre avec l'Autre (Whiteford et Wright St-Clair, 2002 ; Kohli et al., 2010 ; Donoso Brown et al., 2011 ; Hall et Theriot, 2016). En outre, les étudiants racontent comment ils ont acquis des outils d'intervention durant leur stage, que ce soit dans leur manière d'agir ou dans la communication. À nouveau, ces adaptations font partie intégrante de la compétence interculturelle selon plusieurs auteurs (Murden et al., 2008 ; Cai, 2016 ; Hall et Theriot, 2016 ; Reyneke, 2017).

La dernière question posée dans la présente étude concerne les changements perçus par les étudiants dans leur façon d'appréhender les interactions avec des populations diversifiées. À ce propos, des informateurs-clés évoquent la prise de conscience de préjugés et de représentations erronées qui peuvent biaiser l'intervention. Dans ce sens, ils ont expliqué comment la rencontre avec l'Autre leur a permis de briser certains préjugés et de se faire leur propre avis sur les personnes aux références culturelles diverses. Cela s'accorde avec les conclusions de plusieurs écrits scientifiques selon lesquels l'exposition à la diversité constitue un moyen efficace de développer 
une pratique culturellement sensible (Kohli et al., 2010 ; Donoso Brown et al., 2011 ; Hall et Theriot, 2016 ; Reyneke, 2017).

Un autre changement évoqué est l'adoption d'une image plus juste de la profession. Des participants ont relativisé les retombées de leur intervention, et d'autres ont constaté qu'il n'est pas toujours possible de cautionner les décisions de l'Autre. En travail social, Freund et ses collaboratrices (2017) décrivent également cette conception «idéaliste » que les étudiants peuvent avoir de leur futur métier et qui diffère de ce qu'ils vivent une fois sur le terrain.

Un dernier constat lié au processus de transformation est la meilleure aisance dans la pratique en contexte multiculturel que les étudiants disent avoir acquise. Certains illustrent cela par l'adoption d'autres manières d'agir et le recours à des outils d'intervention plus adaptés. Plus confiants, ils insistent sur l'importance d'observer et de s'interroger sur les croyances qui sous-tendent les comportements des personnes qu'ils considèrent comme différentes d'eux. Ces résultats concordent avec les travaux de Murden et ses collaboratrices (2008). Ces dernières se sont intéressées aux changements dans la compétence interculturelle des étudiants en ergothérapie, entre le début de leur formation et leur première année de pratique (incluant les stages). Elles notent que les participants à leur étude souhaitaient être mieux préparés aux aspects interculturels lors de la formation théorique. Les étudiants considéraient néanmoins que le stage permettait d'augmenter leurs connaissances en termes de pratique en contexte de diversité par le contact direct avec des personnes aux cadres de référence différents (Murden et al., 2008). Dans ce sens, Whiteford et Wright St-Clair (2002) énoncent l'importance cruciale des stages dans le développement d'une sensibilité interculturelle et d'une pratique centrée sur la personne. Pour elles, observer comment les professionnels agissent sur le terrain permet aussi d'améliorer les compétences des étudiants. II faut ajouter que l'activité de réflexion sur sa propre pratique, exercice que les participants ont réalisé lors de la présente étude, permet de renforcer les apprentissages faits lors des stages (Doutrich et Storey, 2004 ; Reyneke, 2017).

Le manque d'écrits scientifiques concernant les retombées du stage sur le développement de la compétence interculturelle des étudiants en travail social et en ergothérapie rend difficile la comparaison avec les résultats d'études similaires. En effet, la plupart des travaux consultés évaluent l'efficacité de formations réalisées en classe à l'aide d'une ou de plusieurs techniques (cours, études de cas, discussions de groupe, etc.) (Kohli et al., 2010 ; Donoso Brown et al., 2011 ; Hall et Theriot, 2016 ; Reyneke, 2017). Les apprentissages sur le terrain ne sont pas pris en compte. Le constat est le même notamment pour la médecine, les sciences infirmières et la psychiatrie (Doutrich et Storey, 2004 ; Bhui et al., 2007 ; Dogra et al., 2009). Rares sont les études qui mesurent les effets de la pratique et de la rencontre de personnes aux cadres de référence différents sur le développement de la sensibilité interculturelle des étudiants (Whiteford et Wright St-Clair, 2002 ; Ekelman et al., 2003).

\section{Recommandations pour la formation}

Compte tenu des propos des étudiants et des écrits scientifiques consultés, des recommandations peuvent être formulées. Tout d'abord, il semble important de préparer les étudiants à la rencontre avec l'Autre, en utilisant différents outils pédagogiques (génogramme culturel, activités basées sur l'apprentissage par expérience, vignettes et études de cas, groupes de discussion, etc.) (Donoso Brown et al., 2011 ; Warde, 2012 ; Hall et Theriot, 2016 ; Reyneke, 2017). En outre, l'exposition à la diversité est considérée comme centrale dans la progression vers une pratique culturellement sensible (Kohli et al., 2010 ; Donoso Brown et al., 2011 ; Hall et Theriot, 2016 ; Reyneke, 2017). Celle-ci peut se faire en classe ou lors des stages. Toutefois, les superviseurs ou professionnels 
encadrant les étudiants devraient être davantage formés au contexte multiculturel et aux défis qu'il comporte (Doutrich et Storey, 2004 ; Kohli et al., 2010 ; Hall et Theriot, 2016). Ainsi, ils pourront offrir un cadre sécurisant dans l'apprentissage de la compétence culturelle. De plus, il serait essentiel d'aider les étudiants à prendre conscience des changements qui s'opèrent dans leur pratique suite à la rencontre de l'Autre (Doutrich et Storey, 2004 ; Reyneke, 2017). Pour ce faire, la tenue de journaux de bord, l'utilisation de portfolio ou encore les discussions en groupe peuvent s'avérer efficaces.

Des recherches futures pourraient porter sur les retombées des stages dans le développement de la sensibilité interculturelle des étudiants. Une meilleure connaissance des méthodes de formation les plus adéquates pour l'augmentation des compétences en contexte de diversité devrait être acquise par des recherches plus poussées dans le domaine. II semble aussi intéressant de prendre en compte la satisfaction des personnes accompagnées quant aux services qu'ils reçoivent.

\section{Limites}

Bien qu'innovante, cette étude comporte quelques limites. D'abord, elle s'est déroulée en Suisse romande et il faudrait documenter la situation dans d'autres pays. En outre, l'échantillon interrogé reste limité sur le plan du nombre de participants. Enfin, malgré le fait que la personne qui rencontre les étudiants n'ait aucun lien avec leur formation, il est probable qu'un biais de désirabilité sociale soit apparu, car les questions abordent des sujets sensibles.

\section{Conclusion}

La formation professionnelle des étudiants dans le domaine de la santé et des services sociaux en contexte de diversité nécessite l'intégration de contenus liés aux interactions avec l'Autre. Comme les cadres de référence de chacun (valeurs, croyances, appartenances, etc.) influencent les attitudes et les comportements, il importe de rendre les étudiants attentifs à ces aspects. II est essentiel de leur fournir des outils pour faire face à des valeurs différentes des leurs, savoir réagir adéquatement devant des comportements inattendus, ou encore évoluer dans une variété d'environnements. Le manque de recherches quant aux méthodes optimales de transmission de la compétence interculturelle et au type d'encadrement nécessaire à cet apprentissage met en exergue la nécessité d'explorer ce thème d'actualité en étroite collaboration avec les milieux de formation et professionnels.

\section{REMERCIEMENTS}

Les auteurs tiennent à remercier les étudiants qui ont accepté de participer à cette étude. Ils remercient également le Fonds national suisse de la recherche scientifique (FNS) pour le financement de la recherche longitudinale (2016-2019) intitulée " Le rapport à l'Autre : exploration de son évolution chez les étudiants en travail social et en ergothérapie ».

\section{RÉFÉRENCES}

Audet, G. (2008). " La relation enseignant-parents d'un enfant d'une autre culture sous l'angle du rapport à l'altérité ", Revue des sciences de l'éducation, vol. 34, n² 2, p. 333-350.

Bardin, L. (2013). L'analyse de contenu, Paris, Presses universitaires de France. 
Beagan, B. L. (2015). «Approaches to culture and diversity: A critical synthesis of occupational therapy literature », Canadian Journal of Occupational Therapy, vol. 82, nº 5, p. 272-282.

Bertaux, D. (1997). Les récits de vie, perspective ethnosociologique, Paris, Nathan.

Bhui, K., Warfa, N., Edonya, P., McKenzie, K. et D. Bhugra (2007). " Cultural competence in mental health care: A review of model evaluations », BMC Health Services Research, vol. 7, $n^{\circ} 1$, p. 110.

Bredendiek, M. et B. Krewer (2003). « Le développement de la construction de l'autre culturel. Vers la réflexion anthropologique de l'autrui dans l'interculturel ", Bulletin de l'Association pour la recherche interculturelle, vol. 39, p. 79-99.

Cai, D.-Y. (2016). " A concept analysis of cultural competence », International Journal of Nursing Sciences, vol. 3, n 3 , p. 268-273.

Careau, E. et C. Vallée (2014). «Recherche qualitative et scientificité », dans S. Tétreault et P. Guillez, Guide pratique de recherche en réadaptation, Louvain-la-Neuve, De Boeck Supérieur.

Côté, D. (2014). " La réadaptation au travail des personnes issues de l'immigration et des minorités ethnoculturelles : défis, perspectives et pistes de recherche ", Perspectives interdisciplinaires sur le travail et la santé, vol. $16, \mathrm{n}^{\circ} 2$, p. 1-23.

Dogra, N., Reitmanova, S. et O. Carter-Pokras (2009). «Teaching cultural diversity: Current status in U.K., U.S., and Canadian medical schools », Journal of General Internal Medicine, vol. 25, no Suppl. 2, p. 164-168.

Donoso Brown, E. V., Muñoz, J. P. et J. M. Powell (2011). «Multicultural training in the United States: A survey of occupational therapy programs ", Occupational Therapy in Health Care, vol. $25, n^{\text {os }} 2-3$, p. 178-193.

Doutrich, D. et M. Storey (2004). « Education and practice: Dynamic partners for improving cultural competence in public health », Family and Community Health, vol. 27, n 4, p. 298-307.

Drapeau, M. (2004). "Les critères de scientificité en recherche qualitative », Pratiques psychologiques, vol. $10, \mathrm{n}^{\circ} 1$, p. 79-86.

Ekelman, B., Bello-Haas, V. D., Bazyk, J. et S. Bazyk (2003). «Developing cultural competence in occupational therapy and physical therapy education: A field immersion approach ", Journal of Allied Health, vol. 32, $\mathrm{n}^{\circ}$ 2, p. 131-137.

Fattebert, S., Rüegger, M. et S. Salerno (2005). Agents de la fonction publique aux prises avec la diversité culturelle: quelle formation en matière de prévention des discriminations ?, Berne, Service de lutte contre le racisme.

Fedor, C.-G. (2014). "Stereotypes and prejudice in the perception of the "Other" », Procedia Social and Behavioral Sciences, vol. 149, p. 321-326.

Feize, L. et J. Gonzalez (2018). «A model of cultural competency in social work as seen through the lens of self-awareness », Social Work Education, vol. 37, n 4, p. 472-489. 
Freund, A., Cohen, A., Blit-Cohen, E. et N. Dehan (2017). «Professional socialization and commitment to the profession in social work students: A longitudinal study exploring the effect of attitudes, perception of the profession, teaching, training, and supervision », Journal of Social Work, vol. 17, $n^{\circ} 6$, p. 635-658.

Govender, P., Mpanza, D. M., Carey, T., Jiyane, K., Andrews, B. et S. Mashele (2017). « Exploring cultural competence amongst OT students ", Occupational Therapy International, vol. 4, article $\mathrm{n}^{\circ} 2179781,8 \mathrm{p}$.

Hall, J. C. et M. T. Theriot (2016). «Developing multicultural awareness, knowledge, and skills: Diversity training makes a difference? », Multicultural Perspectives, vol. 18, n 1, p. 35-41.

Kohli, H. K., Huber, R. et A. C. Faul (2010). " Historical and theoretical development of culturally competent social work practice », Journal of Teaching in Social Work, vol. 30, n 3, p. 252-271.

Leanza, Y. (2011). Exercer la pédiatrie en contexte multiculturel. Une approche complémentariste du rapport institutionnalisé à l'Autre, Genève, Georg.

Legault, G. et L. Rachédi (2008). L'intervention interculturelle ( $2^{\mathrm{e}}$ éd.), Montréal, Gaëtan Morin.

Miles, M. B., Huberman, A. M. et J. Saldaña (2014). Qualitative Data Analysis, Thousand Oaks, Sage Publications.

Murden, R., Norman, A., Ross, J., Sturdivant, E., Kedia, M. et S. Shah (2008). "Occupational therapy students' perceptions of their cultural awareness and competency ", Occupational Therapy International, vol. 15, nº 3, p. 191-203.

Reyneke, R. P. (2017). "Apples and pears: Engaging social work students in social dialogue », Research on Social Work Practice, vol. 27, n² 2, p. 239-247.

Tétreault, S., Gulfi, A. et N. Kühne (2016-2019). Le rapport à l'Autre : exploration de son évolution chez les étudiants en travail social et en ergothérapie, Projet FNS, Lausanne, HES-SO Haute École de travail social et de la santé.

Thomas, D. R. (2006). "A general inductive approach for analyzing qualitative evaluation data ", American Journal of Evaluation, vol. 27, $\mathrm{n}^{\circ} 2$, p. 237-246.

Warde, B. (2012). «The cultural genogram: Enhancing the cultural competency of social work students ", Social Work Education, vol. 31, n 5, p. 570-586.

Whiteford, G. et V. Wright St-Clair (2002). «Being prepared for diversity in practice: Occupational therapy students' perceptions of valuable intercultural learning experiences », British Journal of Occupational Therapy, vol. 65, n 3, p. 129-137. 